10 years ESJ

Special edition

\title{
Perspectivas de eCommerce y los Hábitos de Consumo Tras COVID-19
}

\author{
Alejandra Vega Barrios \\ Arlen Cerón Islas \\ Juan Gabriel Figueroa Velázquez \\ Universidad Autónoma del Estado de Hidalgo, Hidalgo México
}

Doi:10.19044/esj.2021.v17n4p112

Submitted: 28 October 2020

Accepted: 23 November 2020

Published: 07 February 2021
Copyright 2021 Author(s)

Under Creative Commons BY-NC-ND

4.0 OPEN ACCESS

Cite As:

Vega Barrios A., Cerón Islas A. \& Figueroa Velázquez J.G. (2021). Perspectivas de eCommerce y los Hábitos de Consumo Tras COVID-19. European Scientific Journal, ESJ, 17(4), 112. https://doi.org/10.19044/esj.2021.v17n4p112

\section{Resumen}

El presente artículo tiene como finalidad estudiar las tendencias de compra en línea dadas las condiciones actuales de aislamiento que se establecieron por la pandemia COVID-19; por lo que se realiza un análisis cualitativo basado en la metodología de la Teoría Fundamentada, con la identificación y categorización de variables sobre los hábitos de consumo y la experiencia de la compra por Comercio Electrónico (eCommerce). Para la construcción de este trabajo se realizaron entrevistas semiestructuradas a consumidores en la ciudad de Pachuca, Hidalgo; quienes hicieron compras por comercio electrónico en el periodo comprendido de marzo a agosto del año 2020, durante el confinamiento. Los resultados obtenidos en esta primera etapa proporcionan información para la incorporación del eCommerce como parte de las estrategias de exposición y venta de productos para el cambio en los hábitos de consumo dada la "nueva normalidad".

Palabras clave: eCommerce, Hábitos De Consumo, Nueva Normalidad 


\title{
Outlook for eCommerce and Consumer Habits After COVID-19
}

\author{
lejandra Vega Barrios \\ Arlen Cerón Islas \\ Juan Gabriel Figueroa Velázquez \\ Universidad Autónoma del Estado de Hidalgo, Hidalgo México
}

\begin{abstract}
The purpose of this article is to study ECommerce trends between conditions of isolation established by the COVID-19 pandemic. The research was done by a qualitative analysis, that is performed based on the Grounded Theory methodology with the identification and categorization of variables on consumption habits and the experience of buying by eCommerce. Semistructured consumer interviews were conducted for the construction of this work in the city of Pachuca, Hidalgo; the customers have made purchases by eCommerce in the period from March to August 2020, during the confinement. The results obtained in this first stage provide information for the incorporation of eCommerce as part of sales strategies products in the change in consumption habits given the "new normal".
\end{abstract}

Keyword: eCommerce, Consumption Habits, New Normal

\section{Introducción}

Ante el desarrollo y expansión de las nuevas tecnologías de la información, en especial en el comercio electrónico ha generado el creciente interés del análisis de tendencias en los hábitos del consumo ante compras en línea.

En 2020, a nivel mundial, negocios de todo tipo, tamaños y giros se han visto afectados por la pandemia del COVID-19. A partir de las decisiones realizadas por las autoridades tanto sanitarias como gubernamentales, para evitar la propagación y contagios han obligado a los propietarios de los negocios a cerrar y buscar otras formas de seguir, adaptados a lo que hoy se ha denominado "La Nueva Normalidad". Sin duda, esto también vino a cambiar nuestra vida y nuestros hábitos de consumo.

Hernández, M. (2020), menciona que hasta el año pasado en México se contabilizaban 61.9 millones de personas que utilizaban el comercio electrónico, así que se estima que después del confinamiento de la pandemia de COVID-19, se incremente a más de 63 millones de usuarios. 


\section{Comercio electrónico}

La Organización para la Cooperación y el Desarrollo Económico (OECD, 2011), define al comercio electrónico como el proceso de compra, venta o intercambio de bienes, servicios e información a través de redes de comunicación.

El comercio electrónico tiene lugar a través de diversas relaciones comerciales, que implican cualquier posible combinación de consumidores (C), empresas (B) o gobiernos (G). Entre ellas se incluyen las clásicas transacciones B2B, que siguen representando la mayor parte del volumen de negocios derivado del comercio electrónico del sector privado, así como las transacciones de empresa a gobierno (B2G). Las transacciones de comercio electrónico involucran cada vez más a los consumidores de forma directa, sobre todo las transacciones de empresa a consumidor (B2C).

Loor et al (2018) menciona que el eCommerce se ha convertido en la estrategia de las organizaciones que generan capacidades de competencia de las gestiones que realizan los comerciantes y los consumidores. Ahora es necesario que los comerciantes conozcan las nuevas costumbres de consumo que se tienen en la actualidad, los mismos que sufren una transformación constante, eCommerce se adapta a cada cambio generado, con la finalidad de satisfacer las necesidades del consumidor, suministrando información sobre productos, precios y segmento del mercado (p. 4).

Ruiz, A. (2015) menciona de igual forma que esta nueva tendencia de comportamiento del proceso venta contempla cuatro tipos: ventas directas de la empresa, venta en línea al menudeo, comunidades de negocios y mercados de subastas en líneas.

De igual forma ha tenido impacto en las formas de pago tales como: transferencia electrónica de fondos (débito y crédito), E-money, tarjetas de prepago y la banca electrónica. En particular según Loor et al (2018), el eCommerce pretende que exista confianza en las transacciones que se realicen de forma online, varias empresas eligen el comercio electrónico, pero existen interrogantes del porque los consumidores a veces no optan por comprar de esta forma, los clientes sienten cierta desconfianza que lo bloquea y provoca que el usuario no se adapte a la nueva tecnología $(\mathrm{p}, 5)$

Con la finalidad de medir la importancia de este tipo de transacciones se observó que de acuerdo con eMarketer Worldwide Retail and eCommerce SalesForcast 2016 -2023, el crecimiento de comercio electrónico a nivel mundial para el 2023 representará el 21\% de las ventas totales Retail en todo el mundo, teniendo una tasa de crecimiento anual compuesta (CAGR) del $19.7 \%$, para una mejor perspectiva ver la figura 1 donde se visualiza el crecimiento de comercio electrónico a nivel mundial (Orozco, D., 2020).

En cuanto a México, en 2019 se le considero como el país con el mayor crecimiento sobre todo en el rubro Retail con un $35.0 \%$ (figura 2) con respecto 
al crecimiento a nivel mundial, Orozco, D (2020a) de la Asociación Mexicana de Venta on-line (AMVO) muestra que el crecimiento de México tiene una participación de mercado (Share) del $4.9 \%$ con respecto al Retail total de la región de Latinoamérica, lo cual lo convierte en líder regional.

\section{CRECIMIENTO DE COMERCIO ELECTRÓNICO ANIVEL MUNDIAL}

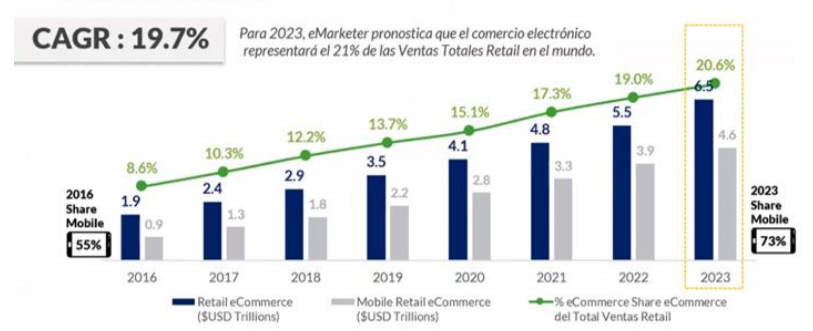

Figura 1. Crecimiento de Comercio Electrónico a nivel mundial.

Fuente: Orozco, D. (2020a)

A 10 años de la penetración del Internet en México, el Instituto de Estadística, Geografía e Informática (INEGI) en su estudio National Survey on Availability and Use of Information Technologies in Households (ENDUTIH) 2019, reporta un 70.1\% de penetración lo que significa que 6 de cada 10 mexicanos tienen acceso a internet, esto representa a 80.6 millones de Internautas.

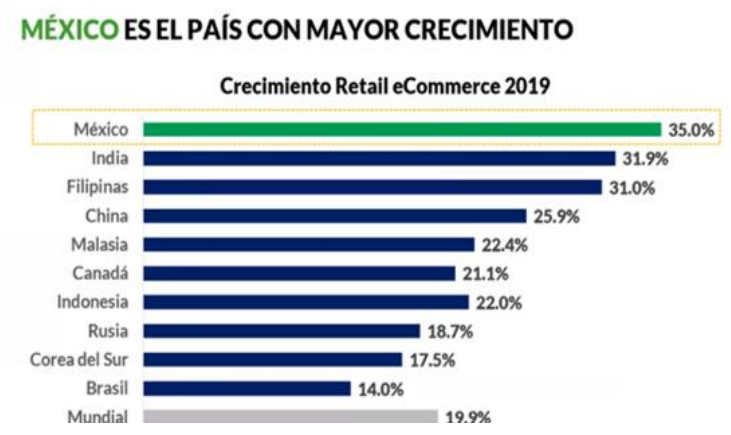

Figura 2. México, País con mayor crecimiento.

Fuente: Orozco, D (2020a)

Bajo este mismo enfoque, Torres, Y. (2020), establece que existe una tendencia de crecimiento alta durante los últimos 3 años, se observa en la figura 3 un incremento del 2017 al 2019 del 63\% alcanzando 631,700 millones de pesos en comercio electrónico en México. 


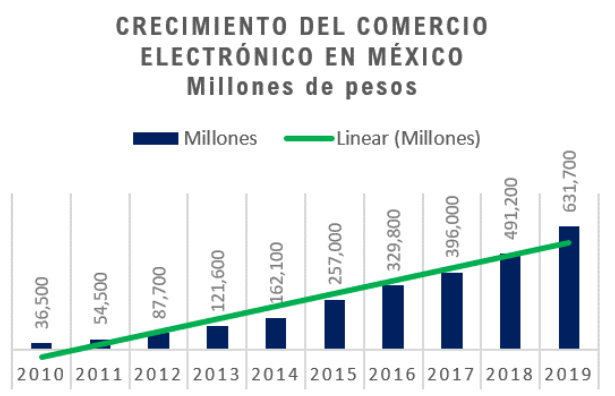

Figura 3. Crecimiento de Comercio Electrónico en México Fuente: Elaboración propia, a partir de Torres, Y. (2020)

Además de la penetración de Internet, es importante revisar la penetración de los teléfonos celulares denominados Smartphones, ya que su evolución también tiene un impacto importante con 102.9 millones de dispositivos, que representan un $87.1 \%$ de líneas móviles de acuerdo con el reporte de Mercado de Smartphones desarrollado por The Competitive Intelligence Unit durante 2018, que vale la pena considerarse debido a que los usuarios ya inician con el comercio electrónico desde su dispositivo.

Con la finalidad de identificar las tendencias de consumo dada la "nueva nomalidad" Statista (2020), ha segmentado el Comercio Electrónico en los diversos rubros, considerando cinco grandes categorías: "comida y cuidado personal"; "muebles y electrodomésticos"; "juguetes y hobbies"; "electrónica de consumo" (tecnología) y "moda" (ropa y calzado); algunos de ellos han resultado beneficiados por considerarse como actividades esenciales; tales como el pago de servicios de plataformas de contenido digital (Over The Top-OTT), con un $23.4 \%$, el pago de servicios a un $16.2 \%$, las compras en super mercado en $13.9 \%$ y las aplicaciones delivery en $11.4 \%$

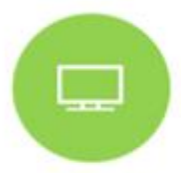

$23.4 \%$

Pago de Servicio or

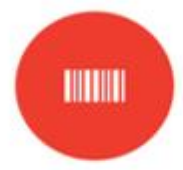

$16.2 \%$

Pago de Servicio

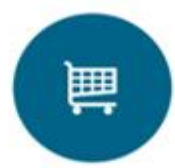

$13.9 \%$

Supermercado

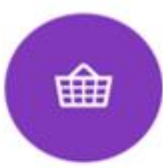

$11.4 \%$

Aplicaciones de Delivery

Figura 4. Actividades esenciales ante la Pandemia COVID -19

Fuente: The Competitive Intelligence Unit, 2020; tomado de Forbes México, 2020

Según Visa Consulting \& Analytics (2020), muestra que las transacciones en línea en América Latina en comparación con las ventas en físico incrementaron 5.8 veces en mayo, al realizar una comparación con los meses de enero y febrero; en este análisis se muestran tres tendencias: aceleración por el eCommerce, priorización de adquisiciones de bienes y 
servicios de primera necesidad y un incremento en transacciones de comercio electrónico (cita en Infobae, 2020).

El estudio de la agencia MRM Madrid (2020), identifica el aumento de consumo de contenidos registrado en las últimas semanas y en las plataformas de streaming como Netflix, HBO o Movistar; de igual forma se observa el aumento del uso de los videojuegos es especialmente notable en la Generación Z y las plataformas de streaming especializadas, como Twitch han incrementado su tasa de interés (búsqueda); también se identifica un incremento en el uso de plataformas de música en streaming; este estudio revela que la venta online de productos de alimentación se ha triplicado; debido a esto se propone que las marcas de gran consumo diseñen una estrategia relacional con el consumidor y recorrer el "path to parchase" con él para llegar a la venta online.

Ruíz, A. (2020), menciona que el consumo en línea se ha consolidado como la única vía de acceso a contenidos informativos, de entretenimiento, culturales y educativos durante el aislamiento, también el pago de servicios ha registrado un marcado crecimiento de 5.9 por ciento, al ser una vía segura y efectiva para cumplir con las obligaciones de pago y así, evitar tanto riesgos de contagio, como de propagación del virus.

Hernández, M. (2020), comenta que los segmentos que más van a destacar en cuanto a su crecimiento en las compras vía online, hasta una demanda del $300 \%$, son aquellas áreas de despensa, farmacia, abarrotes, higiene personal, limpieza, alimentos para mascotas, productos digitales (como música, películas y videojuegos) y servicios de comida; esto por el lado de los comercio y por el lado del consumidor el $69 \%$ de los compradores serán de clase media; planteando que los segmentos de la base de la pirámide son los que no van a aprovechar el comercio digital eso quizás influenciado por la bancarización y el enrolamiento digital de pagos.

Las aplicaciones de entrega de comida a domicilio como Uber Eats, Rappi, Sin Delantal, entre otras, que han permitido y facilitado la continuidad en la operación de restaurantera bajo condiciones estrictas de higiene. El segmento de la población que no se acercaba a este tipo de servicios en línea, ya sea por miedo al robo de datos bancarios, por la falta de conocimiento o práctica en la manipulación de estas plataformas han debido superarse, y durante esta etapa de aislamiento social se traerá una nueva educación digital, tanto para consumidores como para proveedores que han de iniciarse e integrar a esta forma comercial en donde migrarán a tiendas virtuales (Ruiz, A., 2020).

\section{Método}

La presente investigación presenta un estudio de corte cualitativo realizado mediante el enfoque de la Teoría Fundamentada (Glaser y Strauss, 
1967); siendo el objetivo del estudio identificar y categorizar variables sobre los hábitos de consumo y la experiencia de la compra por Comercio Electrónico (eCommerce)

Se realizaron entrevistas semi-estructuradas a consumidores en la ciudad de Pachuca, Hidalgo; que hayan realizado compras electrónicas en el periodo comprendido de marzo a agosto del año 2020; una revisión teórica e histórica de artículos científicos de las tendencias del eCommerce.

\section{Preguntas de Investigación}

Las preguntas de investigación en las que se basó el estudio fueron enfocadas a consumidores de productos alimenticios y servicios mediante eCommerce:

P1. ¿Cómo han cambiado sus hábitos de consumo en productos alimenticios por eCommerce a partir del confinamiento?

P2. ¿Cuál es la experiencia que ha tenido en el uso del eCommerce?

\section{Informantes}

El estudio se llevó a cabo en la ciudad de Pachuca, Hidalgo, dónde participaron personas adultas con experiencia en compras mediante eCommerce. Los criterios de elegibilidad para fueron: tener residencia mínima de 2 años en la ciudad de Pachuca, Hidalgo; edad entre 25 y 45 años, contar con una computadora o dispositivo electrónico para la realización de compras on-line, haber realizado compras por este medio; contar con tarjeta de crédito o débito para la realización de compras y encontrarse en confinamiento.

Para la selección de los informantes, se realizó una entrevista vía telefónica a quince personas para determinar la elegibilidad, seleccionándose diez personas que cumplían con los criterios y que además se comprometieran a participar en el estudio durante el periodo de marzo a agosto del año en curso.

Se elaboró una tabla con la descripción de los participantes seleccionados con sus características para identificar los más representativos para el estudio (ver tabla 1).

Tabla 1. Descripción de los participantes

\begin{tabular}{|c|c|c|c|}
\hline Participante & Sexo & Edad & Ocupación \\
\hline PT1. & F & 33 & Profesora de Primaria \\
\hline PT2. & F & 45 & Enfermera General \\
\hline PT3. & F & 25 & Ejecutiva de ventas \\
\hline PT4. & M & 30 & Contador público \\
\hline PT5. & M & 28 & Estudiante \\
\hline PT6. & M & 44 & Ingeniero Civil \\
\hline PT7. & M & 38 & Arquitecto \\
\hline PT8. & M & 26 & Vendedor de autos \\
\hline PT9. & F & 25 & Estudiante \\
\hline PT10. & F & 30 & Médico Veterinario \\
\hline
\end{tabular}

Fuente: Elaboración propia, 2020 
Para el tamaño de la muestra de este trabajo se empleó el método de muestreo selectivo basado en la Teoría Fundamentada ya que como proceso interpretativo depende de la sensibilidad del investigador a los elementos tácitos de los datos (Glaser y Strauss 1967), las entrevistas fueron analizadas mediante el software denominado Atlas Ti8, el cual permite identificar aquellos códigos que requieren saturación de contenido y categoría, tal como se propone en la Teoría Fundamentada.

\section{Guía de Entrevista}

La entrevista semi estructurada consistió primeramente en preguntas generales de identificación, adentrándose en los temas que se requerían para la obtención de la información, tales como: “¿Cómo son tus hábitos de consumo en cuanto a alimentos?”, “Estos hábitos han cambiado a partir del confinamiento?”, "¿Ha cambiado tu forma de pago en la adquisición de productos?", „¿Cuál ha sido tu experiencia?”

Los datos obtenidos de las entrevistas semi estructuradas abiertas fueron conducidas por el investigador con la finalidad de documentar las experiencias con el uso del eCommerce y los cambios en los hábitos de consumo. La entrevista fue dividida en bloques de preguntas con la finalidad de facilitar el análisis, obteniéndose cinco bloques con diferentes temas (ver tabla 2): 1B. Hábitos de consumo antes de la pandemia, 2B. Hábitos de consumo después de la pandemia, 3B. Sobre eCommerce, 4B. Sobre formas de pago, 5B. Sobre envío de los productos.

Tabla 2. Estructura de la guía de la entrevista

\begin{tabular}{|c|c|}
\hline Bloques de preguntas & Número de preguntas \\
\hline Hábitos de consumo antes de la pandemia & 8 \\
\hline Hábitos de consumo después de la pandemia & 10 \\
\hline Sobre eCommerce & 8 \\
\hline Sobre formas de pago & 6 \\
\hline Sobre envío de los productos. & 8 \\
\hline
\end{tabular}

Fuente: Elaboración propia, 2020

Cada una de las entrevistas tuvo una duración de 45 minutos aproximadamente, las cuales fueron grabadas para ser analizadas en el software Atlas Ti8, codificadas de forma abierta de acuerdo con la Teoría Fundamentada.

\section{Análisis de los Datos}

Para Strauss, et al. (2002), el propósito principal de la Teoría Fundamentada en los datos consiste en generar o encontrar modelos explicativos sobre determinados fenómenos, cuyos principios teóricos se encuentran apoyados en el análisis sistemático y posteriormente a la 
interpretación de los datos obtenidos de un conjunto de informantes mediante entrevistas.

En la aplicación de la teoría fundamentada, la codificación teórica es el principal procedimiento de análisis para construir la teoría. En este procedimiento de interpretación de datos se pueden distinguir tres procesos: 1) codificación abierta, 2) codificación axial y 3) codificación selectiva; que se llevó a cabo mediante la herramienta Atlas Ti8.

\section{Codificación Abierta}

Para realizar la codificación abierta de los datos obtenidos a través de las entrevistas semiestructuradas, tomadas en audio de WhatsApp se analizaron detalladamente las transcripciones y a través del software Atlas Ti8, se identificaron códigos y categorías por medio de la agrupación de conceptos (ver figura 5).

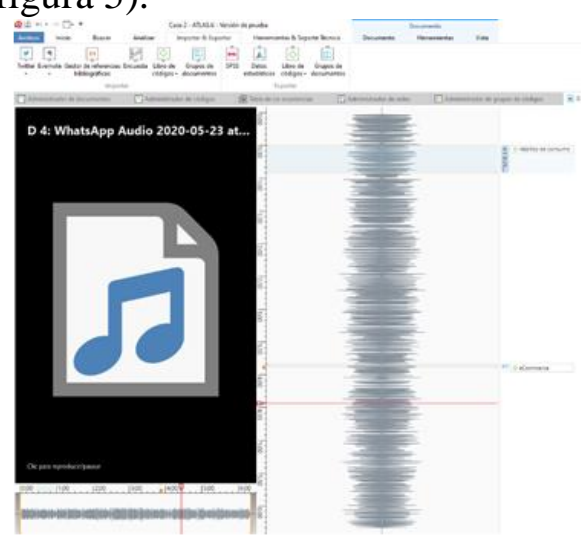

Figura 5. Codificación abierta de datos a través de audio de WhatsApp.

Fuente. Elaboración propia a partir de entrevistas semiestructuradas, 2020.

\section{Codificación Axial}

En la codificación axial se identificaron las relaciones entre las categorías obtenidas en la codificación abierta, en la cual se determinaron la variedad de condiciones, acciones/interacciones y consecuencias asociadas a los hábitos de consumo y el eCommerce. Por lo cual se llevó a cabo la jerarquización en subcategorías que son relacionadas con las categorías obtenidas previamente.

En la figura 6, se muestra los grupos de códigos abiertos de la transcripción de los datos recogidos. 


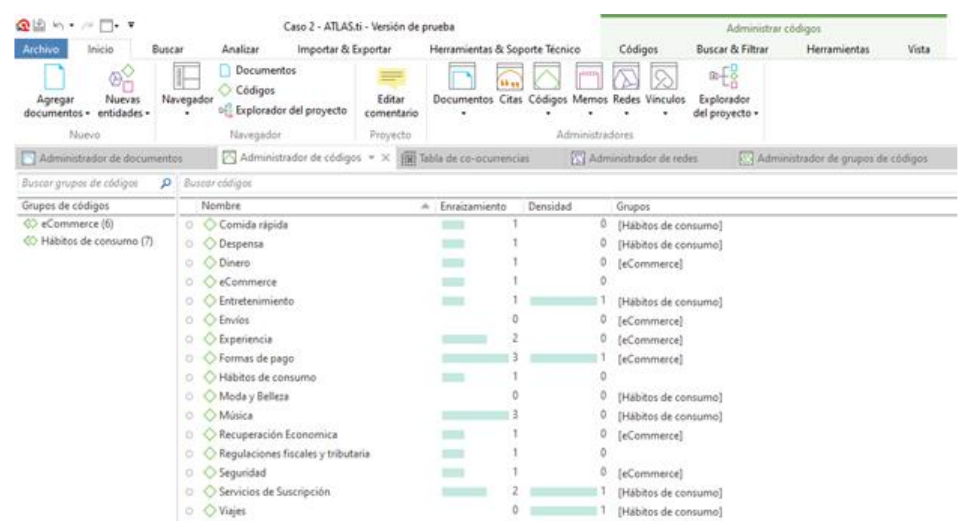

Figura 6. Grupos de Códigos y Codificación abierta de datos, 2020.

Fuente: Elaboración propia a partir de entrevistas semiestructuradas

Estos códigos seleccionados sirvieron posteriormente para la identificación de patrones e información importante para desarrollar una tabla de análisis de las categorías relevantes para el estudio.

Tabla 3. Descripción de categorías, subcategorías, propiedades y dimensiones

\begin{tabular}{|c|c|c|c|}
\hline Categorías & Subcategorías & Propiedades & Dimensiones \\
\hline \multirow{23}{*}{$\begin{array}{l}\text { Hábitos de } \\
\text { Consumo }\end{array}$} & \multirow{15}{*}{$\begin{array}{c}\text { Antes de la } \\
\text { pandemia }\end{array}$} & \multirow{5}{*}{$\begin{array}{l}\text { Frecuencia de } \\
\text { compra }\end{array}$} & Semanal \\
\hline & & & Mensual \\
\hline & & & Bimensual \\
\hline & & & Semestral \\
\hline & & & Anual \\
\hline & & \multirow[t]{3}{*}{ Tipo de compra } & Planeadas \\
\hline & & & Por impulso \\
\hline & & & No planeadas \\
\hline & & \multirow[t]{5}{*}{ Tipo de productos } & $\begin{array}{c}\text { Comida y cuidado } \\
\text { personal }\end{array}$ \\
\hline & & & Entretenimiento \\
\hline & & & Servicios \\
\hline & & & Moda \\
\hline & & & Tecnología \\
\hline & & \multirow[t]{2}{*}{ Ubicación } & Casa \\
\hline & & & Oficina \\
\hline & \multirow{8}{*}{$\begin{array}{c}\text { Después de la } \\
\text { pandemia }\end{array}$} & \multirow{5}{*}{$\begin{array}{l}\text { Frecuencia de } \\
\text { compra }\end{array}$} & Semanal \\
\hline & & & Mensual \\
\hline & & & Bimensual \\
\hline & & & Semestral \\
\hline & & & Anual \\
\hline & & \multirow[t]{3}{*}{ Tipo de compra } & Planeadas \\
\hline & & & Por impulso \\
\hline & & & No planeadas \\
\hline
\end{tabular}




\begin{tabular}{|c|c|c|c|}
\hline & & \multirow[t]{5}{*}{ Tipo de productos } & $\begin{array}{c}\text { Comida y cuidado } \\
\text { personal }\end{array}$ \\
\hline & & & Entretenimiento \\
\hline & & & Servicios \\
\hline & & & Moda \\
\hline & & & Tecnología \\
\hline & & \multirow[t]{2}{*}{ Ubicación } & Casa \\
\hline & & & Oficina \\
\hline \multirow[t]{23}{*}{ eCommerce } & \multirow{9}{*}{$\begin{array}{l}\text { Experiencia en la } \\
\text { compra }\end{array}$} & \multirow{3}{*}{$\begin{array}{l}\text { Proceso de } \\
\text { compra }\end{array}$} & Método de pago \\
\hline & & & Autorización de compra \\
\hline & & & Error de cobro \\
\hline & & \multirow[t]{3}{*}{ Confianza } & Alta \\
\hline & & & Regular \\
\hline & & & Nula \\
\hline & & \multirow[t]{3}{*}{ Seguridad } & Eficiente \\
\hline & & & Verificado \\
\hline & & & Datos cerrados \\
\hline & \multirow[t]{8}{*}{ Formas de pago } & \multirow[t]{4}{*}{ Crédito } & $\begin{array}{c}\text { Transferencia electrónica } \\
\text { SPEI }\end{array}$ \\
\hline & & & Monedero Electrónico \\
\hline & & & Depósito en OXXO \\
\hline & & & Dinero electrónico \\
\hline & & \multirow[t]{4}{*}{ Contado } & $\begin{array}{c}\text { Transferencia electrónica } \\
\text { SPEI }\end{array}$ \\
\hline & & & Monedero Electrónico \\
\hline & & & Depósito en OXXO \\
\hline & & & Dinero electrónico \\
\hline & \multirow[t]{6}{*}{ Envíos } & \multirow[t]{3}{*}{ Nacionales } & Precio accesible \\
\hline & & & Rápido \\
\hline & & & Domicilio \\
\hline & & \multirow[t]{3}{*}{ Internacionales } & Precio accesible \\
\hline & & & Rápido \\
\hline & & & Domicilio \\
\hline
\end{tabular}

Fuente: elaboración propia, 2020.

En la Tabla 3 se muestran las categorías que se definieron para interpretación el estudio de eCommerce y los hábitos de consumo estableciendo las relaciones causales donde generan cambios a partir del antes y después de la pandemia denominada COVID -19.

\section{Codificación Selectiva}

En la codificación selectiva se selecciona una categoría para ser el núcleo, y se relacionan todas las demás categorías con la central. La categoría central en este estudio es eCommerce presentada en la tabla 3. 
Así mismo, se describen los hábitos de consumo directamente con otras dos subcategorías, "antes de la pandemia" y "después de la pandemia", las cuales están directamente relacionadas con la categoría central y explican los cambios a partir del fenómeno COVID-19.

\section{Hallazgos}

En conjunto, las tres categorías ayudaron de manera significativa para la creación del modelo que explica los hábitos de consumo en eCommerce, antes y después de la pandemia COVID - 19 .

\section{Hábitos de Consumo} antes de la Pandemia

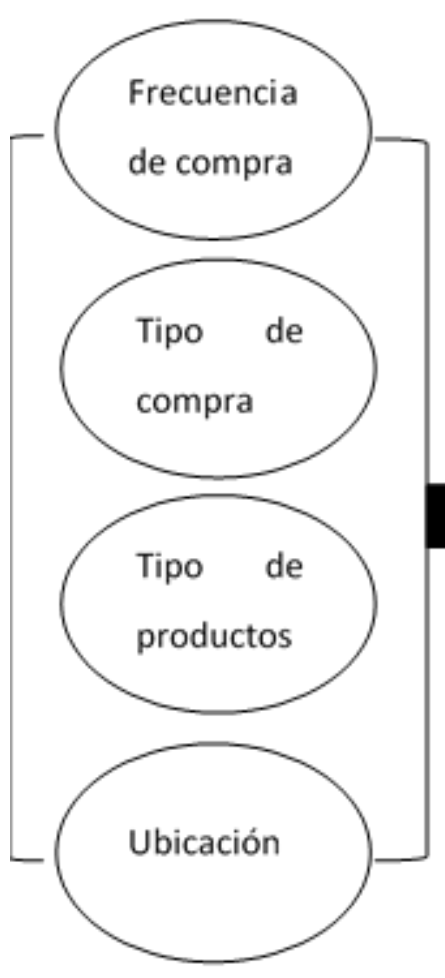

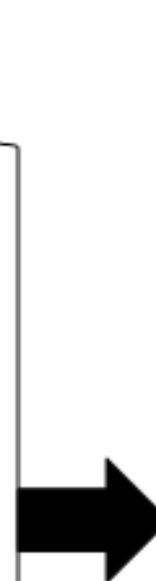

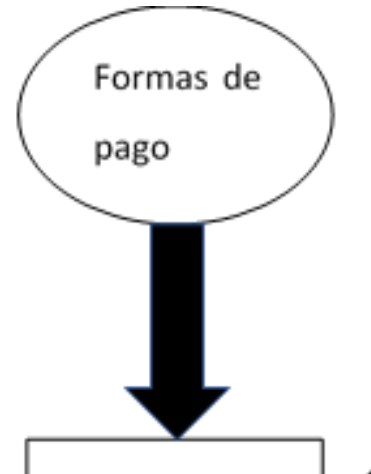

\section{Experiencias}

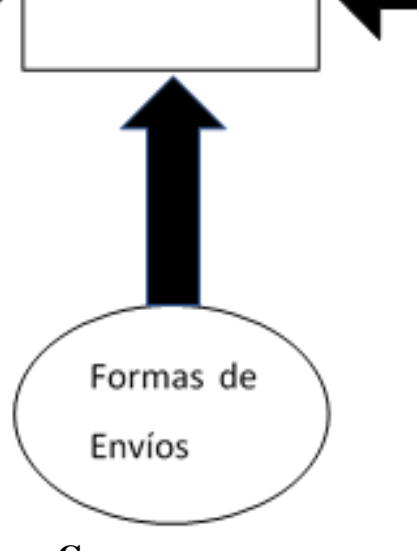

eCommerce

Hábitos de consumo después de la Pandemia

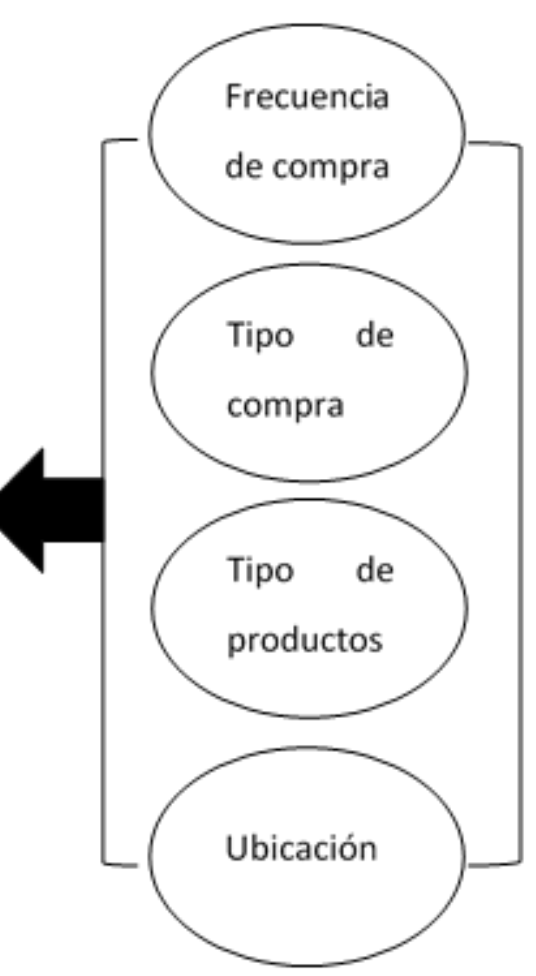

Figura 7. Grupos de Códigos y Codificación abierta de datos.

Fuente. Elaboración propia a partir de entrevistas semiestructuradas

\section{Categoría 1: eCommerce}

Esta categoría es la principal del estudio, en ella se define el eCommerce, a través de las experiencias, determinadas por las formas de pago y envío de los hábitos de compra, influenciadas por las dos subcategorías (antes de la pandemia y después de la pandemia) que preceden y determinan 
el patrón de frecuencia de compra, tipo de compra, tipo de productos y la ubicación desde donde realiza la compra.

\section{Variable 1: Experiencias}

De acuerdo con los comentarios de los participantes se identificó que durante la etapa de confinamiento en la pandemia los consumidores han adquirido productos y servicios mediante una mayor búsqueda de información y procesos de selección que tiene mucho que ver con sus hábitos de consumo sobre qué, dónde y cuánto comprar.

Por lo tanto, los participantes manifestaron que actualmente la experiencia en el uso del eCommerce ha sido con mayor aceptación, pues antes del confinamiento quizá se habrá comprado una o dos ocasiones cuando mucho, sin embargo hoy debemos utilizar y cambiar lo que veníamos consumiendo, como se observa en el comentario del participante siete, en donde expone que después del confinamiento, la experiencia de compra utilizando comercio electrónico; le ha solucionado consumir mejores productos y planear lo más adecuado al bolsillo. Comentario del participante siete (PT7): "Pues desde que estoy en confinamiento, he utilizado más el comercio electrónico para comprar mejores productos que se adaptan a mi presupuesto y si no puedo en el momento, puedo planear y revisar si puedo pagarlo a plazos, lo mejor es que lo hago desde la computadora de mi casa".

\section{Variable 2: Formas de pago}

Las formas de pago, para los participantes denominadas como crédito o de contado, se encontró que los consumidores en este momento requieren mayores opciones que les brinden seguridad al momento de utilizar el eCommerce y establecer la compra para satisfacer sus hábitos de consumo, como se muestra en los siguientes comentarios de los participantes cinco y nueve: "Para tener la seguridad de que te llega lo que compras, es mejor pagar contra entrega como lo hace Walmart, recibes los productos y pagas en el momento, hasta con vales de despensa!" (PT5), para las necesidades básicas siempre se paga de contado, pero para productos grandes si se requiere pagar con crédito y no importa, solo que sea seguro que no te cobren de más" (PT9).

\section{Variable 3: Formas de envío}

En esta variable de formas de envío, se encontró que los consumidores tienen ciertos requisitos para solicitar los envíos nacionales o internacionales, como son que tenga precio accesible o "gratis", que llegue rápido y que sea directo al domicilio como se muestra en los siguientes comentarios de los participantes uno y tres: "Las compras por las páginas internacionales ("asiáticas") usan correos que parecieran ser mexicanos, tardan mucho en la 
entrega y el envío gratis o a un precio accesible" (PT1) "Uso más mercado libre porque casi siempre el envío es gratuito además de la rapidez ..."

\section{Categoría 2: Hábitos de Consumo}

En cuanto a la categoría hábitos de consumo, los participantes comentaron que es un factor importante para la definición de hábito de consumo, debido a que los consumidores pueden considerar la frecuencia con la que realiza la compra (variable 4), el tipo de compra (variable 5), el tipo de producto (variable 6), y la ubicación desde donde se realiza la compra (variable 7) donde cada una de estas variables se explican a continuación.

\section{Variable 4: Frecuencia de compra}

En esta variable sobre frecuencia de compra, antes de la pandemia el hábito de consumo era mensual en clubes de precios, sin embargo, a raíz del confinamiento la frecuencia de compra en productos básicos es semanal y en otro tipo de productos es quincenal, utilizando eCommerce y con entregas inmediatas. como se muestra en los siguientes comentarios de los participantes diez y tres: "Las compras normalmente eran físicas y se hacían en lugares predefinidos en nuestra ciudad, sin embargo, a partir del confinamiento, se ha perdido esta práctica y es por ello que empezamos a utilizar las compras a través de eCommerce, cada semana" (PT10). "Yo compraba solo cada mes, y ahora debo hacerlo semanal y quincenalmente pues los productos básicos deben ser frescos y debo confiar que con esto es suficiente" (PT3).

\section{Variable 5: Tipo de compra}

En esta variable se encontró que actualmente, la realización de compras es planeada a diferencia de como se venían realizando antes de la pandemia. Por otra parte, se debe revisar que, si existe, compras impulsivas y compras no planeadas, debido a que solamente el participante cuatro (PT4) lo mencionó.

\section{Variable 6: Tipo de productos}

Sobre los tipos de productos, se encontró que los participantes han cambiado sus hábitos de consumo en comida a domicilio, moda, belleza y hasta en las compras para las mascotas, así mismo el consumo de entretenimiento como lo es Netflix, como lo comento el participante seis (PT6) "No había utilizado ninguna plataforma para ver películas, y ahora que utilice Netflix, me parece que es maravilloso que pueda adquirir tarjetas para solo tener el control un mes". 


\section{Variable 7: Ubicación}

En esta variable de ubicación, se revisó donde hacen uso del eCommerce siendo que por el confinamiento la mayoría de los participantes lo hace desde su domicilio que ha sido un cambio en los hábitos de consumo, ya que anteriormente la compra la hacían desde su lugar de trabajo.

\section{Discusión}

Ante la denominada nueva normalidad, tanto los consumidores como los negocios se están adaptando y con ello lo que se puede interpretar es que, con la venta de productos por la pandemia COVID-19, acelera el crecimiento del Comercio electrónico, mostrando que las personas cambiarán sus hábitos de consumo y las empresas buscarán la fidelidad a través de los gustos de sus clientes.

En los hábitos de consumo existe una serie de influencias para lograr que las compras se realicen mediante comercio electrónico (eCommerce), quizá en este sentido, lo más importante es el tipo de producto ya que al encontrarse en confinamiento, aquellos productos Como ejemplo de ello, el participante dos opinó "Porque no podemos salir, escogemos lo que se ofrezca mediante internet." (P2), el participante diez comentó "No hay existencia en las tiendas física." (P10) y el participante cinco "a veces en las tiendas no hay lo que buscas y aparte que sale mucho más caro..." (P5); Esta información demuestra que la perspectiva que tiene el eCommerce puede aumentar.

Mientras que los datos de 2018 y 2019 se mantienen al alza por el propio desarrollo y comportamiento del Comercio electrónico y el uso tanto del Internet como los dispositivos móviles, los hábitos de consumo de los internautas habían iniciado con algunos cambios, sin embargo aún existían ciertas inseguridades para poder utilizar de manera frecuente los sitios web que pudieran ofrecer productos mediante la aplicación del Comercio Electrónico, como el ser víctima de fraude electrónico mediante clonación o la extracción de datos personales de tarjetas de crédito, así como el desconocimiento de los métodos de pago y la desconfianza sobre la entrega de los productos adquiridos.

Con la aparición de la pandemia, el comercio electrónico, el uso y penetración del Internet y de los dispositivos móviles; ha tenido un fuerte impacto a nivel mundial, nacional y regional de tal suerte que el crecimiento en los hábitos de compra en línea en los consumidores también han tenido cambios.

Las ventas por Internet en México y en el Estado de Hidalgo se han elevado a través del tiempo, debido a que más empresas están vendiendo y ofreciendo sus productos y servicios de manera online sobre todo a nivel local, ya que los consumidores buscan la conveniencia y comodidad de comprar a 
través portales y páginas que se encuentran en la red o en grupos de compra con la finalidad de reactivar la economía local.

Los principales productos comprados por comercio electrónico (eCommerce) durante la pandemia, han sido comida a domicilio, supermercados, productos para el aseo del hogar, medicamentos y mascotas, los cuales están creciendo debido a la indicación de quedarse en casa, por lo que el comportamiento de gasto a futuro derivado de la pandemia que si existe cambio de hábitos del consumo, ya que de los perfiles seleccionados cinco de los diez consumidores, seguirá comprando en canales de comercio electrónico al finalizar la pandemia, en la ciudad de Pachuca, Hidalgo aunado a las tiendas físicas , brindando esto una buena oportunidad para el comercio electrónico, cuidando las experiencias, a partir de formas de envío y pago.

De esta manera, los datos obtenidos sobre los hábitos de consumo y el eCommerce, demuestran que los consumidores han optado por utilizar con mayor regularidad está nueva opción debido a la situación por la que nos encontramos en este momento, por lo que es importante considerar que el confinamiento apoyará a que estos hábitos cada vez sean más cambiantes, sobre todo en la compra de productos y servicios en los sectores de tecnología, moda y entretenimiento.

\section{Conclusiones}

Los hallazgos de este estudio demuestran que el enfoque metodológico de la Teoría Fundamentada aplicado en el ámbito de Comercio electrónico identifica que los hábitos de consumo cambiaron a partir del confinamiento de la pandemia, los cuales se obtuvieron a partir de una reflexión crítica de la realidad observada.

Con base en los resultados del estudio, se concluye que el análisis de los hábitos de consumo puede ayudar a la creación de estrategias para el comercio electrónico, el cual vislumbra que se tenga inclusión financiera de todos los mexicanos, ya que el $61 \%$ no cuenta con una cuenta bancaria, así como generar confianza en el consumidor ya que el $31 \%$ no se siente seguro al realizar transacciones por internet, la tasa de aceptación de los bancos y la baja de comisiones a las empresas que quieren incursionar en este ámbito.

Las órdenes de quedarse en casa eventualmente se levantarán estado por estado, pero mientras tanto, hay millones de consumidores que crean y refuerzan nuevos comportamientos y hábitos de compra por eCommerce. En muchas familias, las compras realizadas por comercio electrónico en línea de comestibles, ropa y entretenimiento reemplazarán las visitas a tiendas y centros comerciales de forma permanente hasta que haya una vacuna disponible, por lo que la perspectiva del eCommerce es bastante buena. 


\section{References:}

1. Black Sip (2020). Ecommerce en México. 2019 -2020 Reporte de Industria.https://cdn2.hubspot.net/hubfs/1725164/Reporte\%20de\%20 industria\%20del\%20Ecommerce\%20de\%20Mexico\%202019\%20$\% 202020 . p d f$

2. Glaser, B. y A. Strauss. (1967). The Discovery of Grounded Theory: Strategies for Qualitative Research. Aldine Publishing Company. Chicago, Estados Unidos. https://books.google.com.mx/books?hl=es\&lr=\&id=GTMrDwAAQB AJ\&oi=fnd\&pg=PP1\&ots=JtUdICvwfY\&sig=3eu4Tja1oWaWCXYg 1C7SKaY_1ns\&redir_esc $=\mathrm{y} \# \mathrm{v}=$ onepage $\& \mathrm{q} \& \mathrm{f}=$ false

3. Hernández, M. (abril, 2020). El coronavirus acelerará hasta dos años la adopción del e-commerce en México. Forbes México [digital]. https://www.forbes.com.mx/tecnologia-ecommerce-coronavirusadopcion-mexico/

4. INEGI (2016). Información por entidad, Actividades Económicas. Hidalgo.http://cuentame.inegi.org.mx/monografias/informacion/hgo/e conomia/default.aspx?tema $=$ me\&e $=13$

5. INEGI (2019). National Survey on Availability and Use of Information Technologies in Households (ENDUTIH) 2019. http://en.www.inegi.org.mx/programas/dutih/2019/

6. Loor, J. L. M., Navarro, A. D. A., De Lucca, J. B. V., \& Gonzabay, D. E. V. (2018). E-commerce: un factor fundamental para el desarrollo empresarial en el Ecuador. Revista Científica ECOCIENCIA. 5. 1-17.

7. Medina, A. (27 junio, 2020). Comercio electrónico: el 'boom' que llegó para quedarse, pero aún tiene un reto. Forbes México [digital]. https://www.forbes.com.mx/negocios-e-commerce-el-boom-quellego-para-quedarse-pero-aun-tiene-un-reto/

8. MRM Madrid (marzo, 2020). COVID 19 La nueva vida indoor. Marketing directo [Web].

Madrid.https://www.marketingdirecto.com/wpcontent/uploads/2020/03/01_THE-PURPLE-INSIGHTV6_horizontal_def_30_03_20.pdf

9. OECD. (2011). Guide to Measuring the Information Society 2011. OECD Publishing. Paris. https://dx.doi.org/10.1787/9789264113541en

10. Orozco, D. (2020) "Impacto COVID - 19 Reporte 3.0". [webinar] Asociación Mexicana de Venta on line (AMVO). https://www.amvo.org.mx/wpcontent/uploads/2020/07/AMVO_Estudio3.0_ImpactoCOVID19_VP $\%$ C3\%BAblica.pdf 
11. Orozco, D. (2020a) "El panorama del ecommerce y el perfil del comprador online mexicano". [webinar] Asociación Mexicana de Venta on line (AMVO) y Asociación Mexicana de Agencias de Investigación $\quad \mathrm{y} \quad$ Opinión (AMAI). https://www.youtube.com/watch?v=V_JHvB73RpE

12. Ruiz, A. d. 1. Á. R. (2015). Análisis y perspectivas del comercio electrónico en México. Perfiles de las Ciencias Sociales, 3(5).

13. Ruíz, A. d. 1. Á. R. (2020). Emergencia Sanitaria y transacciones electrónicas: COVID-19 Caso México. Perfiles de las Ciencias Sociales, 8(15).

14. Statista (2020) Comercio Electrónico. Estadísticas y datos de mercado sobre comercio electrónico. https://es.statista.com/sectores/1171/comercio-electronico/

15. Strauss, A. L., J. Corbin y E. Zimmerman, (2002) Bases de la Investigación Cualitativa: Técnicas y Procedimientos para Desarrollar la Teoría Fundamentada. Editorial Universidad de Antioquia. Medellín, Colombia. https://diversidadlocal.files.wordpress.com/2012/09/basesinvestigacion-cualitativa.pdf

16. Torres, Y. (13 agosto, 2020) Crecimiento del comercio electrónico en México. Sol de México [digital] https://www.elsoldemexico.com.mx/analisis/crecimiento-delcomercio-electronico-en-mexico-5619098.html

17. TyN Magazine. (27 de mayo 2020). Cómo COVID-19 está transformando el comercio electrónico. https://www.tynmagazine.com/como-covid-19-esta-transformandoel-comercio-electronico/

18. Visa Consulting \& Analytics (2020). Tendencias de consumo muestran repunte en las transacciones en línea en América Latina y el Caribe. Disponible en https://www.visa.com.mx/acerca-de-visa/salade-noticias/notas-de-prensa/tendencias-consumo-transacciones-enlinea.html. Citado en Infobae (Julio, 2020). El comercio electrónico se aceleró en América Latina por la pandemia de COVID-19. México. Infobae [web]. htps://www.infobae.com/america/mexico/2020/07/05/el-comercioelectronico-se-acelero-en-america-latina-por-la-pandemia-de-covid19/ 